\title{
Evaluation of the electrostatic potential, electric field and electric field gradient from the aspherical pseudoatom model in an infinite crystal.$$
\text { J Weatherly }{ }^{1} \text {, A Volkov }{ }^{2} \text {, P Milano }{ }^{3}
$$ \\ ${ }^{1}$ Middle Tennessee State University ${ }^{2}$ MTSU, ${ }^{3}$ No affiliation given \\ jessie.weatherly@mtsu.edu
}

\begin{abstract}
A recently presented method for evaluating the intermolecular electrostatic interaction energies in molecular crystals (Nguyen, D. Macchi, P. \& Volkov, A. (2020) Acta Cryst. A76, 630-651) has been extended to the calculation of the electrostatic potential (ESP), electric field (EF) and electric field gradient (EFG) in an infinite crystal. The proposed technique includes an efficient Ewald-type summation (ES) of atomic multipoles up to the hexadecapolar level in direct and reciprocal spaces, and corrections for the net polarization of the sample due to a net dipole moment of the unit cell, referred to as the surface term (if present), and the short-range electron density penetration effects.

The resulting algorithm was coded using Fortran in the XDPROP module of the XD software package. Testing of the code was performed on several small molecular crystal systems, and the results have been compared to those obtained via direct space summations (DS) performed using an increasing number of unit cells generated in the three spatial dimensions.

The ES-based technique allows for a noticeably more precise determination of the EF and EFG, and significantly better precision of ESP as compared to the DS calculations. This held even when the DS calculations include contributions from a simulated crystal generated with four unit cells along each crystallographic direction. The ESbased technique is also significantly faster than DS calculations performed with the extended crystal, but trails the DS calculations performed using within the reduced summation ranges. However, the proposed ES-based algorithm is superior to the direct space method as the user is not required to continuously monitor the convergence of the calculated properties as a function of the summation limits, and offers a better precision-performance balance.
\end{abstract}

